\title{
Geometry and thermodynamic fluctuations of the Ising model on a Bethe lattice
}

\author{
BY BRIAN P. DOLAN $\dagger$ \\ Physikalisches Institut, Universität Bonn, \\ Endenicher Alle 11-13, D-53115 Bonn, Germany \\ Received 2 July 1997; accepted 11 December 1997
}

A metric is introduced on the two-dimensional space of parameters describing the Ising model on a Bethe lattice of coordination number $q$. The geometry associated with this metric is analysed and it is shown that the Gaussian curvature diverges at the critical point. For the special case $q=2$ the curvature reduces to an already known result for the one-dimensional Ising model. The Gaussian curvature is also calculated for a general ferromagnet near its critical point, generalizing a previous result for $T>T_{\mathrm{c}}$. The general expression near a critical point is compared with the specific case of the Bethe lattice and a subtlety, associated with the fact that the specific heat exponent for the Bethe lattice vanishes, is resolved.

Keywords: Ising model; Bethe lattice; thermodynamics; geometry

\section{Introduction}

The concept of defining a distance function, or metric, on the space of states of a thermodynamic system or statistical distribution has recently been investigated by several authors (for a review from the statistical point of view, see Amari (1985), and from a thermodynamical point of view, see Ruppeiner (1995)). The idea has also been extended to field theories (Zamolodchikov 1986; Jack \& Osborn 1990; O'Connor \& Stephens 1993; Dolan 1997). For two-dimensional field theories, a metric was used to powerful effect in the proof of the c-theorem (Zamolodchikov 1986).

From a thermodynamic and statistical point of view the motivation behind the introduction of a metric lies in a quantitative description of the distinguishability of states: if two states are difficult to distinguish experimentally then they are close to one another in a metrical sense and, conversely, if they are easily distinguished experimentally, then they should be far apart in the metrical sense. In a thermodynamic context, when a system is near a critical point, fluctuations can be large and it can be difficult to distinguish between states; distances then become small and one can expect the curvature associated with any such metric to become large near a critical point.

As described in more detail in the next section, a possible candidate for a metric is associated with the matrix consisting of second derivatives of the free energy. Determining the local geometry, for example, curvature and geodesics, of such a metric would require up to fourth derivatives of the free energy and so perturbation

\footnotetext{
$\dagger$ Currently on leave of absence from Department of Mathematical Physics, St. Patrick's College, Maynooth, Ireland (bdolan@thphys.may.ie).

Proc. R. Soc. Lond. A (1998) 454, 2655-2665

Printed in Great Britain 
theory is of little use in such an endeavour. One is thus led to examine exactly solvable models, with two or more parameters in order to have a non-trivial geometry.

The purpose of this paper is to investigate further the role of a metric in thermodynamics and statistical mechanics by examining in detail a class of exactly soluble models: Ising models on a Bethe lattice of arbitrary coordination number, $q$. Near the critical point, the analysis should not depend in detail on the underlying model, and it is shown how the specific results for the Bethe lattice fit in with a general analysis for a ferromagnet near the critical point.

A metric may be defined on the two-dimensional space of states, which can be parametrized by the spin-spin coupling $J$ and the external field $H$, or by $K=J / T$ and the magnetization per site $M$. Near the critical point the curvature can be expressed purely in terms of critical exponents and the scaling function, provided the specific heat exponent $0<\alpha<1$, and it will be shown how the general result for the curvature relates to that of the Bethe lattice, for which $\alpha=0$. This generalizes the result of Ruppeiner (1995) for the curvature at, and just above, the critical temperature with vanishing magnetization.

In the specific example of the Bethe lattice, with coordination number $q$, it will be shown that the Gaussian curvature is positive and tends to $q / 2$ for high temperature, but diverges at the critical point

$$
K_{\mathrm{c}}=\frac{J}{T_{\mathrm{c}}}=\frac{1}{2} \ln \left(\frac{q}{q-2}\right) .
$$

These results generalize those of the one-dimensional Ising model with periodic boundary conditions derived analytically in Janyszek \& Mrugała (1989), and are significant because they are exact results for a class of models which exhibits a phase transition.

\section{The metric}

As described in the introduction, the motivation for a metric comes from probability theory and the theory of large deviations (Ellis 1985). For example, in thermodynamics (Ruppeiner 1995) two states are considered to be close to one another if the probability of a fluctuation between them is large: if the probability is unity the distance between the states should be zero, if the probability is zero the distance between the states should be infinite. This is satisfied if the distance is a multiple of a positive power of minus the logarithm of the probability. The metric is thus related to the entropy.

Let $g^{a}$ denote the intensive variables (temperature, pressure, external magnetic field, etc.) and $\phi_{a}$ the corresponding extensive variables. The probability of a fluctuation from $\phi_{a}$ to $\phi_{a}^{\prime}$, for given values of $g^{a}$ corresponding to a most probable value $\phi_{a}$, is given by a Gaussian distribution for $\delta \phi_{a}=\phi_{a}^{\prime}-\phi_{a}$ small,

$$
P\left(\phi^{\prime}, g\right)=\mathcal{N} \exp \left\{-S\left(\phi^{\prime}\right)+\frac{1}{2}\left\{\frac{\partial^{2} S(\phi)}{\partial \phi_{a} \partial \phi_{b}}\right\} \delta \phi_{a} \delta \phi_{b}+\ldots\right\}
$$

where $\mathcal{N}$ is a normalization factor and $S$ the thermodynamic entropy (the Einstein summation convention is used throughout).

Taking $-\ln P$ we see that a natural metric is given by

$$
\mathrm{d} l^{2}=-\frac{\partial^{2} S(\phi)}{\partial \phi_{a} \partial \phi_{b}} \mathrm{~d} \phi_{a} \mathrm{~d} \phi_{b}
$$

Proc. R. Soc. Lond. A (1998) 
or, in terms of the Legendre transform to $W=-\ln Z$,

$$
W(g)=-S(\phi)+g^{a} \phi_{a},
$$

with $g^{a}=\partial S / \partial \phi_{a}$,

$$
\mathrm{d} l^{2}=-\frac{\partial^{2} W(g)}{\partial g^{a} \partial g^{b}} \delta g^{a} \delta g^{b} .
$$

We shall work on a lattice with $N$ sites and set $w=W / N=F / N T$, the free energy per site divided by the temperature (we use units in which Boltzmann's constant $k=1$ ). It will then be convenient to rescale the line element (2.4) by the constant factor $1 / N$ and use

$$
\mathrm{d} l^{2}=-\frac{\partial^{2} w}{\partial g^{a} \partial g^{b}} \delta g^{a} \delta g^{b} .
$$

The reason for this rescaling is in order to ensure that the metric makes sense in the thermodynamic limit. If the line element were not rescaled, all distances would be infinite in this limit, since the probability of a fluctuation between two states would always be zero. The curvature associated with the line element (2.5) is finite in general, except possibly at critical points (this will be seen explicitly for the Ising model on a Bethe lattice in the following). Thus the curvature associated with the line element (2.4), which is $1 / N$ times that of (2.5), would always vanish in the thermodynamic limit, except at critical points where it would be ill-defined. This procedure of rescaling quantities by powers of $N$ in order to study their behaviour under fluctuations is standard practice in the theory of large deviations. Thus the interesting geometry and non-zero curvature associated with (2.5) is due to thermal fluctuations.

Equation (2.5) is not covariant under general coordinate transformations, but that is because it is written in a special coordinate system. If $\phi_{a}=\sqrt{N}\left\langle\Phi_{a}\right\rangle$ is the expectation value of the rescaled extensive quantity $\sqrt{N} \Phi_{a}$, then a covariant form of the line element is

$$
\mathrm{d} l^{2}=\left\{\left\langle\Phi_{a} \Phi_{b}\right\rangle-\left\langle\Phi_{a}\right\rangle\left\langle\Phi_{b}\right\rangle\right\} \mathrm{d} g^{a} \mathrm{~d} g^{b},
$$

which reduces to $(2.5)$ when the partition function is the sum over states of the exponential of a linear combination of $\sqrt{N} \Phi_{a}$ with coefficients $g^{a}$,

$$
Z(g)=\sum_{\text {states }} \mathrm{e}^{-\sqrt{N} g^{a} \Phi_{a}} .
$$

The form of equation (2.5) is only preserved under linear transformations of $g^{a}$, not under general coordinate transformations. However, equation (2.6), which reduces to (2.5) when the partition function is of the form (2.7), is general coordinate covariant. In what follows it will be convenient to use the special coordinate system (2.7) in which the metric takes the form,

$$
G_{a b}=-\frac{\partial^{2} w}{\partial g^{a} \partial g^{b}} .
$$

For systems with two variables, e.g. $K=J / T$ and $h=H / T$ with $J$ a spin-spin coupling and $H$ an external magnetic field, it is convenient to perform the Legendre

Proc. R. Soc. Lond. A (1998) 
transform from $(K, h)$ to $(K, M)$ with $M=-\partial w / \partial h$. Changing to these variables, using

$$
G_{a^{\prime} b^{\prime}}=\frac{\partial g^{c}}{\partial g^{a^{\prime}}} \frac{\partial g^{d}}{\partial g^{b^{\prime}}} G_{c d}
$$

in equation (2.8), one finds that the metric in the coordinate system $\left(g^{a^{\prime}}\right)=(K, M)$ is diagonal,

$$
G_{a^{\prime} b^{\prime}}=\left(\begin{array}{cc}
-\Gamma_{K K} & 0 \\
0 & \Gamma_{M M}
\end{array}\right)
$$

where

$$
\Gamma(K, M)=w+h M \quad \text { and } \quad \Gamma_{K K}:=\frac{\partial^{2} \Gamma}{\partial K^{2}} \quad \Gamma_{M M}:=\frac{\partial^{2} \Gamma}{\partial M^{2}} .
$$

Equations (2.10) and (2.11) provide the basis of the calculations in the following two sections.

\section{Curvature of the Bethe lattice}

The Ising model on a Bethe lattice, of coordination number $q$, has partition function

$$
Z=\sum_{\{\bar{\sigma}\}} \exp \left[K \sum_{(i, j)} \sigma_{i} \sigma_{j}+h \sum_{i} \sigma_{i}\right],
$$

where $\sum_{\{\bar{\sigma}\}}$ means a sum over all spin configurations on the lattice and $\sum_{(i, j)}$ is a sum over nearest-neighbour pairs, $K=J / T$ is the spin-spin coupling, and $h \stackrel{(i, J)}{=} H / T$ an external magnetic field. A good reference for a description of the properties of this model is Baxter (1982), whose notation is used here. For sites deep inside the lattice the free energy per site, $f$, is given by

$$
\begin{aligned}
f / T=-\frac{1}{2} q K-\frac{1}{2} q \ln \left(1-z^{2}\right)+\frac{1}{2} \ln \left[z^{2}+1-z(\right. & \left.\left.+x^{-1}\right)\right] \\
& +\frac{1}{2}(q-2) \ln \left(x+x^{-1}-2 z\right),
\end{aligned}
$$

where $z=\mathrm{e}^{-2 K}$ and $x$ is defined implicitly by

$$
x=\frac{\mathrm{e}^{-2 K}+\mathrm{e}^{-2 h} x^{q-1}}{1+\mathrm{e}^{-2 h-2 K} x^{q-1}},
$$

with $0<x<\infty$. This model has a phase transition at $T_{\mathrm{c}}$ given by $K_{\mathrm{c}}=J / T_{\mathrm{c}}=$ $\frac{1}{2} \ln [q /(q-2)]$. The magnetization per site is

$$
M=\frac{\mathrm{e}^{2 h}-x^{q}}{\mathrm{e}^{2 h}+x^{q}},
$$

which ranges from -1 for $x \rightarrow \infty$ to +1 for $x=0$. A derivation of equations (3.2)(3.4) starting from (3.1) can be found in Baxter (1982). Explicitly, equations (3.3) and (3.4) give

$$
x(M, z)=\frac{\sqrt{1-\left(1-z^{2}\right) M^{2}}+z M}{(1+M)}=\frac{1}{x(-M, z)} .
$$

Proc. R. Soc. Lond. A (1998) 
The metric (2.10) is diagonal if the variables $K$ and $M$ are used, so consider the Legendre transform $\Gamma=f / T+M h$,

$$
\begin{aligned}
\Gamma(K, M)=\frac{1}{2} q \ln \left[\frac{2 z^{2}}{\left(1-z^{2}\right)}\right]+\frac{1}{2} q & \ln \left(\frac{s-z}{1-M^{2}}\right)+\frac{1}{2} \ln \left(\frac{1-M^{2}}{4}\right) \\
& +\frac{1}{2} q M \ln \left(\frac{s+z M}{1+M}\right)+\frac{1}{2} M \ln \left(\frac{1+M}{1-M}\right),
\end{aligned}
$$

where $z=\mathrm{e}^{-2 K}$ and $s=\sqrt{1-\left(1-z^{2}\right) M^{2}}$. Equation (3.5) ensures that $\Gamma(K, M)=$ $\Gamma(K,-M)$. The derivation of equation (3.6) from equation (3.2) can be performed by using equations (3.3) and (3.4) to deduce

$$
\begin{aligned}
& \left.\frac{\partial x}{\partial M}\right|_{K}=-\frac{x\left(x+x^{-1}-2 z\right)^{2}}{2\left\{2-z\left(x+x^{-1}\right)\right\}} \\
& \left.\frac{\partial x}{\partial K}\right|_{M}=\frac{2 z x\left(x-x^{-1}\right)}{\left\{2-z\left(x+x^{-1}\right)\right\}} .
\end{aligned}
$$

As described in the introduction, the metric adopted here involves the second derivatives of $\Gamma$ :

$$
G=\left(\begin{array}{cc}
-\Gamma_{K K} & 0 \\
0 & \Gamma_{M M}
\end{array}\right)
$$

One finds

$$
\left.\begin{array}{c}
\Gamma_{M M}=\frac{(y-2 z)^{2}}{4}\left[\frac{1}{\left(1+z^{2}-z y\right)}+\frac{q}{(z y-2)}\right], \\
\Gamma_{K K}=8 q\left[\frac{z^{2}}{\left(1-z^{2}\right)^{2}}+\frac{z}{(y-2 z)(z y-2)}\right],
\end{array}\right\}
$$

where

$$
y=x+x^{-1}=2 \frac{\sqrt{1-\left(1-z^{2}\right) M^{2}}-z M^{2}}{\left(1-M^{2}\right)} .
$$

The Gaussian curvature can be determined from the metric (3.9) by using (3.10). The algebra is tedious, but the final result is remarkably simple:

$$
\mathcal{R}=\frac{\left(1-z^{2}\right)}{2} \frac{\left\{2\left(1+z^{2}-y z\right)-q\left(3 z^{2}-2 z y+1\right)\right\}}{\left\{(z y-2)+q\left(1+z^{2}-y z\right)\right\}^{2}} .
$$

This can be written explicitly as a function of $M$ and $K$, with $z=\mathrm{e}^{-2 K}$ and $s=$ $\sqrt{1-\left(1-z^{2}\right) M^{2}}$,

$$
\mathcal{R}(K, M)=\frac{\left(1-z^{2}\right)\left(1-M^{2}\right)}{2} \frac{\{(q-2)(3 z-s)+4 z\}}{(s-z)\{(q-2)(z-s)+2 z\}^{2}} .
$$

For $T \rightarrow \infty(K=0)$ this reduces to

$$
\mathcal{R}(0, M)=q / 2,
$$

and for $M^{2}=1$

$$
\mathcal{R}(K, 1)=q / 2 .
$$

Proc. R. Soc. Lond. A (1998) 


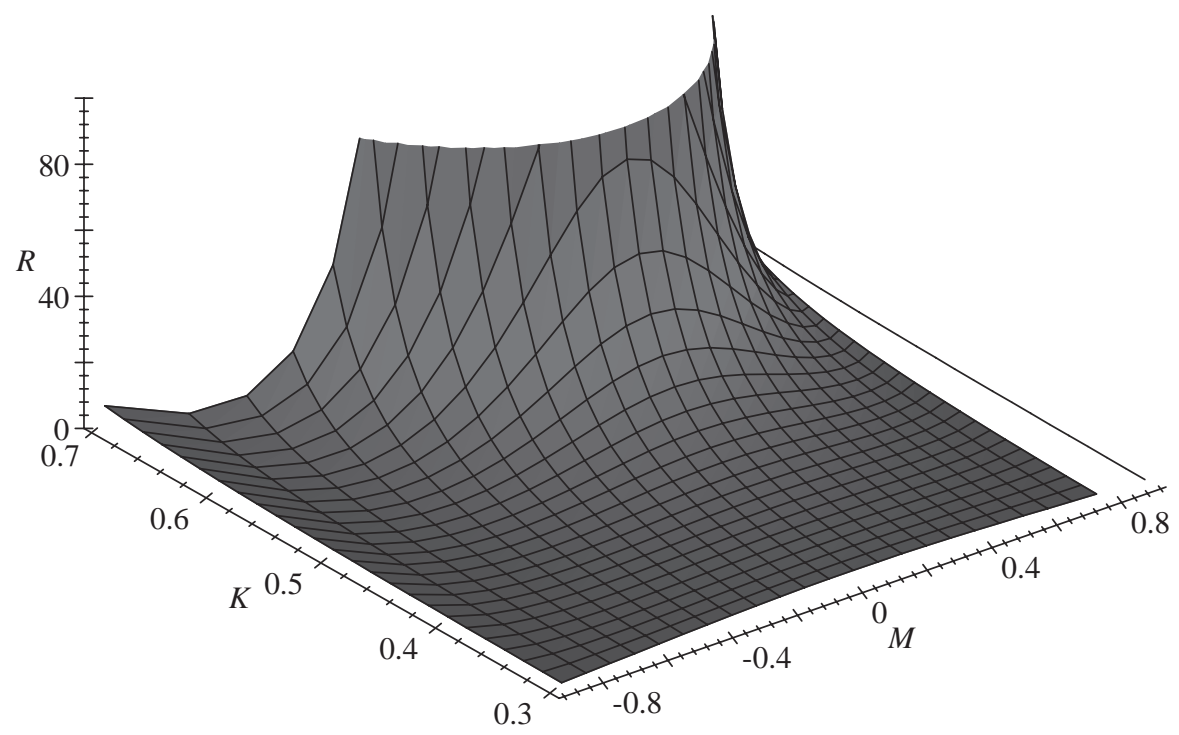

Figure 1. Gaussian curvature for the Ising model on a Bethe lattice (coordination number $q=3$ ). The curvature diverges at the critical point $K=(1 / 2) \ln (3) \approx 0.5493, M=0$, but is finite everywhere else in the stable region of the $K-M$ plane. The line of spontaneous magnetization corresponds to the left-hand edge of the graph (the vertical axis has been truncated at $\mathcal{R}=100$ ).

The curvature is plotted in figure 1 for $q=3$; higher $q$ give qualitatively similar pictures.

Near the critical point, $T=T_{\mathrm{c}}(1+t)$ with $M \ll 1$ and $t \ll 1, z$ is of the form $z=z_{\mathrm{c}}\left\{1+2 K_{\mathrm{c}} t+o\left(t^{2}\right)\right\}$ with

$$
K_{\mathrm{c}}=\frac{1}{2} \ln \left(\frac{q}{q-2}\right) \quad \text { and } \quad z_{\mathrm{c}}=\frac{q-2}{q} .
$$

One then finds

$$
\mathcal{R} \approx \frac{1}{2} \frac{(q-1)^{2}}{q^{2}(q-2)} \frac{1}{\left\{\frac{1}{2} \ln \left(\frac{q}{q-2}\right) t+\left(\frac{q-1}{q^{2}}\right) M^{2}\right\}^{2}} .
$$

In particular, $\mathcal{R}$ diverges to $+\infty$ at the critical point $(M=0, t \rightarrow 0)$.

The line of spontaneous magnetization for $t<0$ is given by the smallest solution of

$$
z=x \frac{\left(1-x^{q-2}\right)}{\left(1-x^{q}\right)}
$$

(see Baxter 1982), and $\mathcal{R}$ is finite along this line, except at the critical point $M=$ $t=0$. For small negative $t$, equation (3.16) gives the equation for the spontaneous magnetization:

$$
M^{2}=(-t) \frac{3 q^{2}}{2(q-1)} \ln \left(\frac{q}{q-2}\right)+o\left(t^{2}\right)
$$




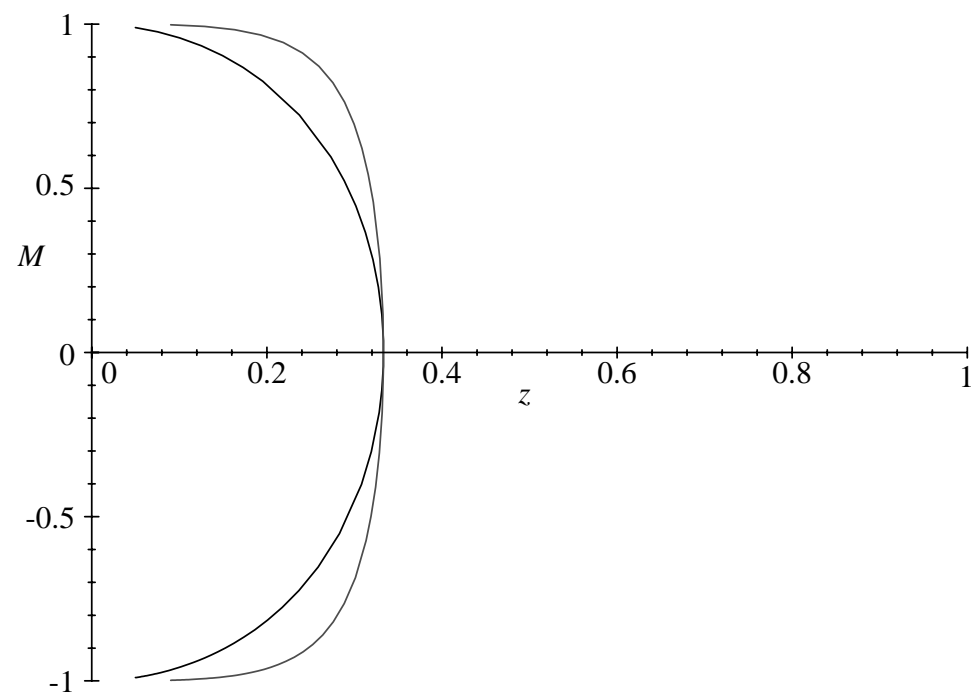

Figure 2. Comparison of the line of spontaneous magnetization and the pseudo-spinodal line for the Ising model on a Bethe lattice with coordination number $q=3$. The vertical axis is the magnetization $M$ and the horizontal axis is $z=\mathrm{e}^{-2 K}$. The leftmost (thick) line is the pseudo-spinodal curve, along which the susceptibility diverges (this lies in the unstable region), the rightmost (thin) line is the line of spontaneous magnetization, which corresponds to the left-hand edge of the graph in figure 1 . These two lines kiss at the critical point $M=0, z=1 / 3$.

Together with equation (3.15) this determines the Gaussian curvature along the line of spontaneous magnetization as

$$
\mathcal{R}=\frac{1}{2} \frac{(q-1)^{2}}{q^{2}(q-2)} \frac{1}{\left\{(-t) \ln \left(\frac{q}{q-2}\right)\right\}^{2}}+o\left(\frac{1}{t}\right),
$$

which is finite except at the critical point $t=0$.

The curvature diverges along the line on which the magnetic susceptibility diverges (the pseudo-spinodal curve (Gaunt \& Domb 1970)). This line lies in the unstable region and does not coincide with the line of spontaneous magnetization (except when $q \rightarrow \infty$ ), though they touch at the critical point. These two curves are plotted in figure 2 for the case $q=3$.

These results are in complete agreement with the general analysis in Ruppeiner (1995). Equation (3.12) reduces to the known result for the one-dimensional Ising model where $q=2$ and $M=\sinh h / \sqrt{\sinh ^{2} h+\mathrm{e}^{-4 K}}$ (Janyszek \& Mrugała 1989).

\section{Curvature near the critical point}

Near the critical point the 'effective action' for a general ferromagnet,

$$
\Gamma(t, M)=f / T+M h,
$$

is assumed to have the scaling form, independent of the microscopic model,

$$
\Gamma=|t|^{2-\alpha} F(x)+\text { analytic part, }
$$

Proc. R. Soc. Lond. A (1998) 
where $x=t / M^{1 / \beta}$ is a scaling variable and $F(x)$ a scaling function, $\alpha$ and $\beta$ are standard critical exponents (see, for example, Zinn-Justin 1993). In the variables $t$ and $M$, the metric is diagonal

$$
G=\left(\begin{array}{cc}
-\frac{\partial^{2} \Gamma}{\partial t^{2}} & 0 \\
0 & \frac{\partial^{2} \Gamma}{\partial M^{2}}
\end{array}\right)
$$

Thus $\left(-G_{t t}\right)$ is the specific heat and $G_{M M}$ the inverse magnetic susceptibility.

In terms of the scaling function, $F(x)$,

$$
\left.\begin{array}{rl}
G_{t t} & =-|t|^{-\alpha}\{(2-\alpha)(1-\alpha) F+(3-2 \alpha) \dot{F}+\ddot{F}\}, \\
G_{M M} & =\frac{|t|^{2-\alpha-2 \beta}}{\beta^{2}} x^{2 \beta}(\beta \dot{F}+\ddot{F}),
\end{array}\right\}
$$

where $\dot{F}=x \mathrm{~d} F / \mathrm{d} x$, etc. Stability requires $G_{t t}>0$ and $G_{M M}>0$, which puts conditions on the function $F$,

$$
\left.\begin{array}{c}
(2-\alpha)(1-\alpha) F+(3-2 \alpha) \dot{F}+\ddot{F}<0, \\
x^{2 \beta}(\beta \dot{F}+\ddot{F})>0 .
\end{array}\right\}
$$

In order that the internal energy $U=-\partial \Gamma / \partial t$ be finite at $t=0$, we must have $\alpha<1$, thus $(2-\alpha)(1-\alpha)>0$. For $x>0$ eliminating $\ddot{F}$ from (4.5) requires

$$
\frac{(3-2 \alpha-\beta)}{(2-\alpha)(1-\alpha)} \dot{F}<F \text {. }
$$

The calculation of the Gaussian curvature from equations (4.4) is straightforward but tedious. The final result can be succinctly expressed by introducing a 'dual' scaling function $\mathcal{F}(x)=x^{2-\alpha} F(x)$. The result is

$\mathcal{R}=\frac{1}{4|t|^{2-\alpha}} \frac{(\alpha+\beta-1)}{(\beta \dot{F}+\ddot{F})(\ddot{\mathcal{F}}-\dot{\mathcal{F}})}\left[\frac{(1-\alpha)\left(\dddot{\mathcal{F}} \dot{\mathcal{F}}-\ddot{\mathcal{F}}^{2}\right)}{(\ddot{\mathcal{F}}-\dot{\mathcal{F}})}-\frac{(2-\alpha-\beta)\left(\dddot{F} \dot{F}-\ddot{F}^{2}\right) x^{2-\alpha}}{(\beta \dot{F}+\ddot{F})}\right]$.

This extends the result of Ruppeiner (1995) to the case of non-zero magnetization, and so is valid for $t<0$ as well as $t>0$ (for comparison, note that the result of Ruppeiner (1995) is quoted in terms of a different function, $\left(1 / T_{\mathrm{c}}\right) Y\left(M / t^{\beta}\right)=$ $\left.F\left(t / M^{1 / \beta}\right)\right)$. The derivation of equation (4.7) assumes that the $|t|^{-\alpha}$ term in $G_{t t}$ (equation (4.4)) dominates over the analytic part, i.e. it assumes that $\alpha>0$, so it is only valid for $0<\alpha<1$. This means that equation (4.7) cannot be applied directly to the Bethe lattice at the critical point, for which $\alpha=0$.

The scaling function, $F(x)$ in equation (4.2), for the Bethe lattice can be obtained by Taylor expanding $\Gamma(K, M)$ in equation (3.6) about $M=0$ and extracting the coefficient of $t^{2}$. First expand $\Gamma(K, M)$,

$$
\begin{aligned}
\Gamma(K, M)=\frac{1}{2} & q \ln \left(\frac{z^{2}}{1+z}\right)+\frac{1}{2}(q-1) \ln 2 \\
& +\frac{M^{2}}{2 !}\left\{1-\frac{1}{2} q(1-z)\right\}+\frac{M^{4}}{4 !}\left\{2-\frac{1}{2} q(z+2)(1-z)^{2}\right\} \\
& +\frac{M^{6}}{6 !}\left\{24-\frac{3}{2} q(1-z)^{3}\left(3 z^{2}+9 z+8\right)\right\}+o\left(M^{8}\right) .
\end{aligned}
$$

Proc. R. Soc. Lond. A (1998) 
Now let $t=\left(T-T_{\mathrm{c}}\right) / T_{\mathrm{c}}$, so that $z=\mathrm{e}^{-2 K_{\mathrm{c}} /(1+t)}$, where

$$
K_{\mathrm{c}}=\frac{1}{2} \ln \left(\frac{q}{q-2}\right)
$$

giving

$$
\Gamma=D+E t+t^{2} F(x)+o\left(t^{3}\right),
$$

where the scaling function has the form (also derived in Baxter (1995))

$$
F(x)=a+\frac{b}{x}+\frac{c}{x^{2}},
$$

and we have defined the following constants:

$$
\left.\begin{array}{rl}
D= & \frac{1}{2} q \ln \left\{\frac{(q-2)^{2}}{q(q-1)}\right\}-\ln 2, \quad E=\frac{q}{2(q-1)}(3 q-2) K_{\mathrm{c},} \\
\begin{array}{l}
a \\
=
\end{array} & -\frac{1}{4} \frac{q K_{\mathrm{c}}}{(q-1)^{2}}\left\{2(3 q-2)(q-1)+q(q-2) K_{\mathrm{c}}\right\}, \\
b & =\frac{1}{2}(q-2) K_{\mathrm{c}}, \\
c & =\frac{(q-2)(q-1)}{12 q^{2}},
\end{array}\right\}
$$

(remember $x=t / M^{1 / \beta}$ ). The subtlety here is that when $\alpha$ is not strictly positive, (4.3) is not quite correct because it assumes that the non-analytic term $1 / t^{\alpha}$ dominates over the analytic part, which is not true when $\alpha=0$. In general one has

$$
\begin{aligned}
G_{t t} & =\left(\frac{\mathrm{d} K}{\mathrm{~d} t}\right)^{2} G_{K K}=-\frac{\partial}{\partial t}\left(\frac{\mathrm{d} K}{\mathrm{~d} t} \frac{\partial \Gamma}{\partial K}\right)+\frac{\mathrm{d}^{2} K}{\mathrm{~d} t^{2}} \frac{\partial \Gamma}{\partial K} \\
& =-\Gamma_{t t}+\left\{\frac{\mathrm{d}^{2} K}{\mathrm{~d} t^{2}}\left(\frac{\mathrm{d} K}{\mathrm{~d} t}\right)^{-1}\right\} \Gamma_{t} .
\end{aligned}
$$

For $\alpha>0, \Gamma_{t t}$ dominates over the second term on the right-hand side, but for the Bethe lattice $\alpha=0$, so the second term must be retained. Taylor expanding $K=K_{\mathrm{c}} /(1+t)$ gives

$$
G_{t t}=-\Gamma_{t t}-2 \Gamma_{t}+o(t)
$$

Using (4.11), (4.10) and (4.12) with $x=t / M^{2}$ gives ( $\beta=\frac{1}{2}$ for the Bethe lattice)

$$
\begin{aligned}
G_{t t} & =-2\left(a+\frac{b}{x}+\frac{c}{x^{2}}\right)-\frac{q}{(q-1)}(3 q-2) K_{\mathrm{c}}+o(t) \\
& =-2\left(\tilde{a}+\frac{b}{x}+\frac{c}{x^{2}}\right)+o(t)
\end{aligned}
$$

where

$$
\tilde{a}:=-\frac{1}{4} \frac{q^{2}(q-2)}{(q-1)^{2}} K_{\mathrm{c}}^{2}=-\frac{b^{2}}{12 c} .
$$

The whole effect of having $\alpha=0$ for the Bethe lattice can thus be absorbed into a redefinition of the constant $a$ in the scaling function ((4.10) and (4.12)). Equation (4.7) can now be applied, with

$$
F(x)=\tilde{a}+b / x+c / x^{2}
$$

Proc. R. Soc. Lond. A (1998) 
to obtain the scaling form of the Gaussian curvature on the Bethe lattice as

$$
\begin{aligned}
\mathcal{R} & =-\frac{b^{2}}{8 \tilde{a} t^{2}} \frac{1}{(b+6 c / x)^{2}}+o\left(\frac{1}{t}\right) \\
& =\frac{(q-1)^{2}}{2 q^{2}(q-2)\left\{K_{\mathrm{c}} t+\frac{(q-1)}{q^{2}} M^{2}\right\}^{2}}+o\left(\frac{1}{t}\right)
\end{aligned}
$$

in agreement with equation (3.15).

\section{Conclusions}

In this paper we have explored the geometrical concept of a metric on the space of states of a thermodynamic system, two states are defined as being far apart if the probability of a fluctuation between them is small. In particular, a class of models which exhibits a phase transition, Ising models on a Bethe lattice, has been examined.

Using expectation values to define a metric on the space of states, as in equation (2.6), the geometry of the Ising model on a Bethe lattice with coordination number $q$ has been analysed and the Gaussian curvature is given by equation (3.12), which is the first main result of this paper. This reduces to the previously known result for the one-dimensional Ising model (Janyszek \& Mrugała 1989) when the coordination number $q=2$. The curvature is seen to be positive definite for all temperatures and magnetizations and diverges at the critical point, but is finite at all other points (in particular, at all other points along the curve of spontaneous magnetization). The Gaussian curvature tends to the constant value $q / 2$ for $T \rightarrow \infty$ as well as for $M= \pm 1$ (a particular case of the latter situation is, of course, $T \rightarrow 0$; for $T>0$ it can only be obtained in the limit of the external field $h$ going to infinity).

For a general ferromagnetic the scaling form of the Gaussian curvature near the critical point has been calculated in terms of the scaling function and its derivatives, (4.7), which generalizes the $t>0, M=0$ result of Ruppeiner (1995). This is our second main result. It has been shown how this relates to the scaling form of the Gaussian curvature on the Bethe lattice, equation (3.12).

An interesting open problem associated with the above analysis is to ascertain whether or not there is a relationship between the renormalization flows for the Ising model on a Bethe lattice and the geometry described here. For the special case of the one-dimensional Ising model, it is already known that the renormalization flow from $T=\infty$ to $T=0$, along the $M=0$ axis, is a geodesic and that this is the only renormalization trajectory that is a geodesic (Dolan 1997). For the more general case with $q>2$, it is certainly still true that the $M=0$ axis is a geodesic-this follows from the observation that the metric (and curvature) are even functions of $M$ and thus invariant under a change in sign of $M$-and this axis will be a line of renormalization flow, but it is an open question as to whether or not any other renormalization trajectories are geodesics for the $q>0$ case. This question merits further investigation.

The author acknowledges support from the Alexander von Humboldt foundation as well as the hospitality of the Physikalisches Institut in Bonn, where the manuscript was completed. Some sponsorship was also received from Baker Consultants Ltd, Ireland, networking specialists (http://ww. baker.ie).

Proc. R. Soc. Lond. A (1998) 


\section{References}

Amari, S. 1985 Differential geometric methods in statistics. Lecture Notes in Statistics, vol. 28. Springer.

Baxter, R. J. 1982 Exactly solved models in statistical mechanics. New York: Academic Press.

Dolan, B. P. 1997 Int. J. Mod. Phys. A 12, 2413-2424.

Ellis, R. S. 1985 Entropy, large deviations and statistical mechanics. Grundlehren der Mathematischen Wissenschaften, vol. 271. Springer.

Gaunt, D. S. \& Domb, C. 1970 J. Phys. C 3, 1442.

Jack, I. \& Osborn, H. 1990 Nucl. Phys. B 343, 647.

Janyszek, H. \& Mrugała, R. 1989 Phys. Rev. A 39, 6515.

O'Connor, D. \& Stephens, C. R. 1993 Geometry, the renormalisation group and gravity. In Directions in general relativity (ed. B. L. Hu, M. P. Ryan Jr \& C. V. Vishveshwava). Proc. 1993 Int. Symp., Maryland, vol. 1. Cambridge University Press.

Ruppeiner, G. 1995 Rev. Mod. Phys. 67, 605.

Zamolodchikov, A. B. 1986 Pis'ma Zh. Eksp. Teor. Fiz. 43, 565.

Zinn-Justin, J. 1993 Quantum field theory and critical phenomena, 2nd edn. Oxford University Press. 
